УДК 539.3

\title{
ДИНАМИКА ГЕОМЕТРИЧЕСКИ И ФИЗИЧЕСКИ НЕЛИНЕЙНОГО ЧУВСТВИТЕЛЬНОГО ЭЛЕМЕНТА НАНОЭЛЕКТРОМЕХАНИЧЕСКОГО ДАТЧИКА В ВИДЕ НЕОДНОРОДНОЙ НАНОБАЛКИ, НАХОДЯЩЕЙСЯ В ТЕМПЕРАТУРНОМ И ШУМОВОМ ПОЛЯХ
}

\author{
Крысько Вадим Анатольевич1, \\ tak@san.ru
}

\author{
Папкова Ирина Владиславовна 1,2 , \\ ikravzova@mail.ru
}

Яковлева Татьяна Владимировна1,2,
yan-tan1987@mail.ru

\author{
Захарова Алёна Александровна3,2, \\ zaa@tu-bryansk.ru
}

\author{
Жигалов Максим Викторович1, \\ zhigalovm@yandex.ru
}

Крысько Антон Вадимович1,2, anton.krysko@gmail.com

1 Саратовский государственный технический университет им. Ю.А. Гагарина, Россия, 410054, г. Саратов, ул. Политехническая, 77.

2 Национальный исследовательский Томский политехнический университет, Россия, 634050, г. Томск, пр. Ленина, 30.

3 Брянский государственный технический университет,

Россия, 241035, Россия, г. Брянск, бул. 50 лет Октября, 7.

\begin{abstract}
Актуальность исследования. Наноэлектромеханические системы, будучи высокочувствительными датчиками, имеющими малье размеры, и надежными в эксплуатации, находят все более широкое применение в нефртегазовой промьшленности для мониторинга различных процессов в нефртедобыче - от разведки до повышения нефртеотдачи, а также при бурении скважин, очистке, фрракционировании и переработке до вывода их из эксплуатации. Одним из примеров применения наноэлектромеханических систем является сейсмические исследование месторождений. Применение наноэлектромеханических систем позволяет улучшить производительность в дополнение к существенной экономии средств и времени для широкого спектра технологий нефтегазовой промьшиленности. Благодаря возможности непрерывного контроля эти технологии могут стать основой «умных» месторождений.

Цель: построение математической модели, наиболее полно описывающей нелинейную динамику чувствительного элемента наноэлектромеханического датчика под действием знакопеременной нагрузки. Для этого необходимо учесть наиболее распространённые в настоящее время кинематические гипотезы, масштабные эффректы с помощью моментной теории упругости, нелинейную зависимость между напряжениями и деформациями, неоднородность материала, шумовые и тепловые поля. А также исследовать характер сложных нелинейных колебаний и выявить закономерности перехода их от гармонических к хаотическим.

объекты: геометрически и физически нелинейная нанобалка, описываемая кинематической моделью первого приближения, на которую воздействует равномерно распределенная знакопеременная поперечная нагрузка с гармонической составляющей, температурное поле и аддитивный внешний шум.

Методы: вариационные методы, метод конечных разностей второго порядка точности для сведения системы нелинейных дисфференциальных уравнений в частных производных к задаче Коши, метод Ньюмарка для решения задачи Коши, метод переменных параметров упругости Биргера для решения физически нелинейной задачи, метод вариационных итераций для получения аналитического решения двумерного уравнения теплопроводности.

Результаты. Для получения аналитического решения теплопроводности применяется метод вариационных итераций. Построена математическая модель колебаний чувствительного элемента наноэлектромеханического датчика в виде размерно-зависимой балки, на которую действует равномерно распределенная поперечная нагрузка с гармонической составляющей. Помимо переменной нагрузки учитывалось влияние температурного поля и аддитивного внешнего шумового воздействия. Геометрическая нелинейность принята по теории Теодора фон Кармана (связь между деформациями и перемещениями). Для учета физической нелинейности материала балки применяются деформационная теория пластичности и метод переменных параметров упругости. Уравнения движения элемента механической системы, а также соответствующие граничные и начальные условия выведены исходя из принципа Остроградского-Гамильтона на базе модифицированной моментной теории с учетом гипотезы Эйлера-Бернулли. Выявлено, что температурное и шумовое поля уменьшают нагрузку, при которой происходит переход в хаотическое состояние системы. Переход от гармонических колебаний к хаотическим происходит по сценарию Рюэля-Такенса-Ньюхауза.
\end{abstract}

\section{Ключевые слова:}

Нанобалка Эйлера-Бернулли, наноэлектромеханическая система, акселерометр для измерения параметров буровых скважин, температурное и шумовое поля, модифрицированная моментная теория упругости, метод конечных разностей и Ньюмарка, хаотические колебания наноэлектромеханической системы.

\section{Введение}

Наноэлектромеханические (НЭМС)-устройства обладают уникальными свойствами, которые определяют их актуальность для практического применения.
Среди свойств можно выделить такие, как низкая масса, высокая электрическая прочность, высокие частоты механического резонанса, потенциально большие квантовые механические эффекты, большое отношение поверхности к объему, что важно для чув- 
ствительных элементов некоторых типов датчиков, например датчиков давления. Наносенсоры (кантиливеры, нановесы, резонаторы и др.) и наноактюаторы (наномоторы, шестеренки и т. д.) используются в физике, биологии, химии, медицине (диагностика, клеточная нано- и микрохирургия, доставка лекарств в пораженное место организма), электронной и нефтегазовой промышленности.

Условия эксплуатации НЭМС датчиков в большей степени совпадают с аналогичными для микроэлектромеханических датчиков (МЭМС). Это температурные воздействия в широком диапазоне от -40 до $150{ }^{\circ} \mathrm{C}$, электростатические поля [1], наличие вибрационных $[2,3]$ и шумовых воздействий [4]. Но в связи со сверхмалыми размерами НЭМС датчиков эти возмущающие факторы будут оказывать на НЭМС устройства более значительное воздействие, чем на МЭМС [5-7].

Наноэлектромеханические системы находят широкое применение в экономически выгодных отраслях нефтегазовой промышленности, таких как нефтедобыча и нефтепереработка. Технологическое оборудование современного нефтеперерабатывающего производства представляет собой комплекс аппаратов машин и вспомогательных устройств, составными частями которых являются балки, подвергающиеся различным динамическим нагрузкам. Так, например, балки и стержни являются составными элементами в колоннах насоснокомпрессорных труб и бурильных колонн, используемых при скважинной нефтедобыче [8-10]. «Сердцем» нано- и микроэлектромеханических датчиков является чувствительный элемент (ЧЭ), от качеств которого зависит точность самого датчика. Такие характеристики датчиков, как прочность, точность, стойкость к высокой температуре (постоянная рабочая температура во время работы в скважине достигает $150{ }^{\circ} \mathrm{C}$, при этом возможны скачки и до более высоких температур) и внешним воздействиям (при бурении возникают случайные динамические воздействия, называемые в механике шумовыми), чрезвычайно важны при создании приборов геофизического назначения, оборудования для нефтегазового и топливно-энергетического комплекса. Следует отметить, что изменения температурного поля негативно влияют на точность измерений, а постоянные внешние случайные (шумовые) вибрации приводят к разрушению измерительных ячеек в приборах, и это может привести к возникновению резонансных колебаний, а впоследствии - к ложным показаниям или даже разрушению прибора.

В настоящее время НЭМС датчики приходят на смену МЭМС устройствам, условия эксплуатации этих приборов во многом сходны, поэтому при их разработке необходимо учитывать тепловые и вибрационные воздействия на составные компоненты [11-14]. Также при исследовании динамики компонентов НЭМС, в отличие от МЭМС, необходимо учитывать неоднородность (гетерогенность) материала и, следовательно, зависимость его физических свойств от деформации, температуры, пространственных координат и времени.
Таким образом, создание методики расчета и проектирования механических компонентов НЭМС является актуальной задачей.

Составляющими механическими элементами НЭМС являются балки, пластины и оболочки, находящиеся под действием случайных - шумовых - воздействий и температурных полей. При построении математических моделей этих механических структур, в частности балочных, необходимо учитывать влияние их размеров на характер колебаний. Зависимость упругого поведения от размеров тела в микронном масштабе экспериментально наблюдалась в разных веществах (металлах [15] и сплавах [16], полимерах [17], кристаллах [18], биомембранах [19]). Экспериментальные наблюдения являются одним из методов моделирования и изучения наноразмерных структур, но из-за дорогостоящего характера этого метода используются другие методы, такие как методы молекулярной динамики, и подходы, основанные на механике сплошных сред, которые обходятся дешевле, с точки зрения вычислений, чем методы молекулярной динамики.

В начале 1900-х гг. братья Коссера [20] предложили теорию для изучения поведения наноматериалов. Их теория явилась началом неклассических теорий для изучения механического поведения наноструктур. Некоторые другие неклассические теории для изучения поведения наноструктур - это моментная теория, теория градиента деформации, теория поверхностных напряжений, нелокальная теория. В моментной теории в энергии деформации рассматривается только вектор градиента вращения, и поэтому требуются только два параметра шкалы длины материала. Модифицированная моментная теория была предложена Ф. Янгом и др. [21] на основе моментной теории. При учете условия равновесия моментов для обеспечения симметричности тензора парных напряжений (моментов) количество параметров шкалы длины материала модифицированной моментной теории уменьшается с двух до одного.

Одним из важнейших аспектов использования моментной теории упругости является ее приложение для задач статики и динамики балок. Для построения математических моделей балок используются гипотезы Бернулли-Эйлера, Тимошенко, ШереметьеваПелеха [22] и др. Каждую из гипотез, лежащих в основе математической модели, можно рассматривать с точки зрения приближения балки от одномерного к двумерному и трехмерному телу. Наиболее популярным приближением является математическая модель на основе гипотез Бернулли-Эйлера. В работах $[23,24]$ с помощью модифицированной моментной теории упругости получены определяющие линейные уравнения, начальные и граничные условия размернозависимой модели Эйлера-Бернулли. Исследовано влияние размерного параметра на статическое деформирование и величину собственных частот. Для статической задачи рассматривается линейное уравнение 4-го порядка для прогиба. Используется метод Бубнова-Галеркина в первом приближении, сводящий уравнение в частных производных к обыкновен- 
ному дифференциальному уравнению по времени. В работе [25] получены уравнения для геометрически нелинейной задачи балки Эйлера- Бернулли на основе соотношений Теодора фон Кармана. Для получения численного решения использован метод БубноваГалеркина в первом приближении. Было проведено исследование влияния размерного коэффициента на величину собственной частоты нелинейных колебаний.

В достаточно малых масштабах физическое поведение материалов становится зависимым от размера, что может влиять на механические, термические, электрические и магнитные характеристики конструкций и механических компонентов [26, 27]. Следовательно, прогресс в области микромеханики и наномеханики и связанных с ними технологий должен идти рука об руку с разработкой усовершенствованных моделей, зависящих от размера, для физических явлений, таких как термо- и электромеханика. В зависящей от размера термоупругости [28] температура может создавать дополнительную термическую деформацию искривления (кручения) в дополнение к обычной деформации термической деформации. S.L. Soboley [29] и D.Y. Tzou [30] также предположили, что теплопроводность в микро/нано масштабе по существу нелокальна и классический закон теплопроводности должен быть дополнительно усовершенствован путем введения характеристической длины материала.

Несмотря на большое количество предложенных новых моделей теплопроводности, теория Фурье также активно продолжает использоваться. Например, в работе [31] представлены основные переходные уравнения и (безразмерные) скорости волн для изотропных и ортотропных тел, а также для изотермических и термоупругих случаев. Для ортотропного твердого тела распространение происходит в главной плоскости. В термоупругих случаях рассматриваются закон Фурье и тепловая релаксация.

Исследованию фазовых переходов, индуцируемых шумом, в науке и технике уделяется большое внимание. Обобщение этих результатов приведено в монографии [32], где рассмотрено влияние шума в задачах физики, химии и биологии. В задачах радиофизики этому вопросу посвящены работа авторов [33] и ряд других. Исследования влияния шумовых нагрузок на динамику балок пластин и оболочек представлены в работах [34-36].

Проведенный авторами анализ показывает, что в известной литературе не описаны модели и исследования размерно-зависимого термоупругого поведения нанобалок, находящихся под воздействием шумовых полей, а также с учетом неоднородности материала при решении задач с двумя типами нелинейности (геометрической и физической). Поэтому важной проблемой до настоящего времени остается построение математических моделей наномеханических систем с учетом действия полей температуры и внешних случайных воздействий. Этому направлению посвящена настоящая работа.

\section{Постановка задачи}

Рассмотрим двумерную область $\Omega$ пространства $\mathrm{R}^{2}$ с декартовой системой координат, введенной следующим образом: в теле балки фиксируется линия приведения, называемая срединной линией $z=0$, ось $\mathrm{OX}$ направлена слева направо вдоль срединной линии, ось OZ - вниз, перпендикулярно ОХ. В декартовой системе координат балка как двумерная область $\Omega$ определяется следующим образом:

$$
\Omega=\left\{x \in[0, a] ; y \in[0, b] ;-\frac{h}{2} \leq z \leq \frac{h}{2}\right\}, 0 \leq t \leq \infty .
$$

Предполагается, что материал нелинейно-упругий и изотропный. Область находится в постоянном температурном поле $T(x, z)$. Расчетная модель и граничные условия приведены на рис. 1 . На $\Gamma_{1}, \Gamma_{3}$ принято граничное условие - жесткая заделка, $\Gamma_{2}$ свободна от нагрузок. По границе $\Gamma_{4}$ приложена вертикальная нагрузка $q=q_{0} \sin \left(\omega_{p} t\right)$, направленная вниз.

Используются следующие гипотезы: тело балки упругое, изотропное, но неоднородное; геометрическая нелинейность вводится по модели Теодора фон Кармана; соотношения получены на базе модифицированной моментной теории упругости с учетом модели Эйлера-Бернулли.

$$
\mathrm{q}=\mathrm{q}_{\mathrm{o}} \sin \left(\omega_{\mathrm{p}} \mathrm{t}\right) \quad \Omega
$$

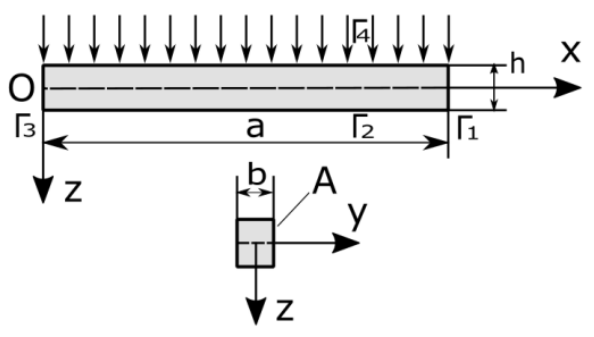

Рис. 1. Расчетная схема

Fig. 1. Design scheme

Для поля смещений $(x, z)$ уравнение равновесия имеют вид

$$
\sigma_{i j}=0 \text { в } \Omega \text {, }
$$

где $\sigma_{i j}-$ тензор напряжений. Связь линейных деформаций с перемещениями определяется по соотношениям

$$
\varepsilon_{i j}=\frac{1}{2}\left(u_{i, j}+u_{j, i}\right), i, j=1,2 .
$$

Соотношение напряжение-деформация записывается по закону Дюгамеля-Неймана [37]

$$
\begin{aligned}
& \sigma_{i j}=(\lambda(x, y, z)+2 \mu(x, y, z)) \times \\
& \times\left(\varepsilon_{i j}-\alpha_{t}(x, y, z) T(x, y, z) \delta_{i j}\right),
\end{aligned}
$$

где $\lambda(x, y, z), \mu(x, y, z), \alpha_{t}(x, y, z), T(x, y, z)$ обозначают параметры Ламе, коэффициент температуропроводности неоднородного материала, температура балки и $\delta_{i j}$ - символ Кронекера, соответственно. Поля смещений и температуры связаны через уравнение (1).

На основании модифицированной моментной теории запасенная энергия упругой деформации балки $U$ с учетом ее размерно-зависимого поведения может быть записана как 


$$
U=\frac{1}{2} \iint_{\Omega}\left(\sigma_{i j} \varepsilon_{i j}+m_{i j} \chi_{i j}\right) d \Omega,
$$

где $\sigma_{i j}, \varepsilon_{i j}, m_{i j}$ и $\chi_{i j}$ обозначают компоненты симметричной части тензора напряжений $\sigma$, тензора деформаций $\varepsilon$, девиаторной части симметричного тензора момента высшего порядка $m$ и симметричной части тензора кривизны $\chi$, соответственно.

Поле смещений в произвольной точке нанобалки Эйлера-Бернулли записываются в виде

$$
u_{1}=u(x, t)-z \frac{\partial w(x, t)}{\partial x}, u_{2}=0, u_{3}=w(x, t),
$$

где $u(x, t), w(x, t)$ обозначают осевое перемещение и поперечное отклонение точек срединной линии, то есть при $z=0$.

Используя (3), нелинейные соотношения Теодора фон Кармана могут быть выражены через поле смещений как:

$$
\varepsilon_{x x}=\frac{\partial u}{\partial x}+\frac{1}{2}\left(\frac{\partial w}{\partial x}\right)^{2}-z \frac{\partial^{2} w}{\partial x^{2}} .
$$

Также из равенства $\phi_{i}=\frac{1}{2}(\operatorname{rot}(\mathbf{u}))_{i}$ можно записать

$$
\phi_{x}=0, \phi_{y}=-\frac{\partial w}{\partial x}, \phi_{z}=0 .
$$

Отсюда получаем следующее выражение для единственного неравного нулю компонента симметричной части тензора кривизны как

$$
\chi_{x y}=\chi_{y x}=-\frac{1}{2} \frac{\partial^{2} w}{\partial x^{2}} .
$$

Определяющие соотношения при наличии температурного поля для неравного нулю компонента девиаторной части тензора момента высшего порядка имеет вид:

$$
m_{x y}=l^{2} \mu \chi_{x y},
$$

где $l$ - материальный размерно-зависимый параметр длины. Размерно-зависимый параметр - это константа, определяющая физические свойства материала, полученная экспериментальным путем. Эта константа появляется в математической модели, если материал рассматривается как континуум Коссера со стесненным вращением частиц. Вместе с модулем Юнга и коэффициентом Пуассона показывает связь между компонентами тензора изгиба кручения и компонентами тензора моментов высших порядков.

Из уравнений (2) и (3)-(7) может быть записана энергия деформации $U$ неоднородной нанобалки как

$$
\begin{aligned}
& U=\frac{1}{2} \int_{0}^{L} \int_{A}\left(\sigma_{x x} \varepsilon_{x x}+2 m_{x y} \chi_{x y}\right) d A d x= \\
& =\frac{1}{2} \int_{0}^{L} \int_{A}\left\{\begin{array}{l}
\sigma_{x x}\left(\frac{\partial u}{\partial x}+\frac{1}{2}\left(\frac{\partial w}{\partial x}\right)^{2}\right)- \\
-\sigma_{x x} z \frac{\partial^{2} w}{\partial x^{2}}+2 m_{x y}\left(-\frac{1}{2} \frac{\partial^{2} w}{\partial x^{2}}\right)
\end{array}\right\} d A d x= \\
& =\frac{1}{2} \int_{0}^{L}\left\{N_{x}\left(\frac{\partial u}{\partial x}+\frac{1}{2}\left(\frac{\partial w}{\partial x}\right)^{2}\right)-M_{x} \frac{\partial^{2} w}{\partial x^{2}}+2 Y\left(-\frac{1}{2} \frac{\partial^{2} w}{\partial x^{2}}\right)\right\} d x,(8)
\end{aligned}
$$

где $A$ - площадь поперечного сечения балки, нормальная результирующая сила $N$, изгибающий момент $M$ и момент высшего порядка $Y$ имеют вид:

$$
\begin{gathered}
N_{x}=\int_{A} \sigma_{x x} d A=k_{1}\left(\frac{\partial u}{\partial x}+\frac{1}{2}\left(\frac{\partial w}{\partial x}\right)^{2}\right)-k_{2} \frac{\partial^{2} w}{\partial x^{2}}-N_{t}, \\
M_{x}=\int_{A} \sigma_{x x} z d A=k_{2}\left(\frac{\partial u}{\partial x}+\frac{1}{2}\left(\frac{\partial w}{\partial x}\right)^{2}\right)-k_{3} \frac{\partial^{2} w}{\partial x^{2}}-M_{t}, \\
Y=\int_{A} m_{x y} d A=-k_{4} \frac{\partial^{2} w}{\partial x^{2}}, N_{t}=\int_{A}(\lambda+2 \mu) \alpha_{t} T d A, \\
M_{t}=\int_{A}(\lambda+2 \mu) \alpha_{t} T z d A .
\end{gathered}
$$

Кинетическая энергия балки $K$ определяется выражением:

$$
\begin{gathered}
K=\frac{1}{2} \int_{0}^{L} \int_{A} \rho\left\{\left(\frac{\partial u}{\partial t}-z \frac{\partial^{2} w}{\partial x \partial t}\right)^{2}+\left(\frac{\partial w}{\partial t}\right)^{2}\right\} d A d x= \\
=\frac{1}{2} \int_{0}^{L} \int_{A} \rho\left\{\left(\frac{\partial u}{\partial t}\right)^{2}-2 z \frac{\partial u}{\partial t} \frac{\partial^{2} w}{\partial x \partial t}+z^{2} \frac{\partial^{2} w}{\partial x \partial t}+\frac{\partial^{2} w}{\partial t^{2}}\right\} d A d x= \\
=\frac{1}{2} \int_{0}^{L}\left\{b_{0}\left(\frac{\partial u}{\partial t}\right)^{2}-2 b_{1} \frac{\partial u}{\partial t} \frac{\partial^{2} w}{\partial x \partial t}+b_{2} \frac{\partial^{2} w}{\partial x \partial t}+b_{0}\left(\frac{\partial w}{\partial t}\right)^{2}\right\} d x .
\end{gathered}
$$

Компоненты жесткости и инерционные члены в уравнениях (9) и (10) определяются как

$$
\begin{gathered}
\left(k_{1}, k_{2}, k_{3}\right)=\int_{A}(\lambda+2 \mu)\left(1, z, z^{2}\right) d A, \\
k_{4}=\int_{A} \mu l^{2} d A,\left(b_{0}, b_{1}, b_{2}\right)=\int_{A} \rho\left(1, z, z^{2}\right) d A .
\end{gathered}
$$

Вариация работы $\delta W$ произведенной внешней силой $q$ имеет вид:

$$
\delta W=\int_{0}^{L} q \delta w d x .
$$

Уравнения движения неоднородной размернозависимой балки Эйлера-Бернулли могут быть выведены из принципа Гамильтона

$$
\int_{0}^{t}(\delta K-\delta U+\delta W) d t=0 .
$$

Подставляя (8), (10) и (11) в (12) и интегрируя по частям, получим разрешающие уравнения движения гибких неоднородных нанобалок с учетом упрощений и стационарного температурного поля, аддитивного белого шума и поперечной знакопеременной нагрузки: 


$$
\begin{gathered}
\frac{\partial}{\partial x}\left(C_{0}(x) \frac{\partial u}{\partial x}+C_{0}(x) \frac{1}{2}\left(\frac{\partial w}{\partial x}\right)^{2}-C_{1}(x) \frac{\partial^{2} w}{\partial x^{2}}\right)-\frac{\partial N_{t}}{\partial x}=\frac{\partial^{2} u}{\partial t^{2}} \\
\frac{\partial^{2}}{\partial x^{2}}\left(C_{1}(x) \frac{\partial u}{\partial x}+C_{1}(x) \frac{1}{2}\left(\frac{\partial w}{\partial x}\right)^{2}-C_{2}(x) \frac{\partial^{2} w}{\partial x^{2}}\right)-\frac{\partial^{2} M_{t}}{\partial x^{2}}+ \\
+L_{1}(u, w)+\frac{1}{2} L_{2}(w, w)-L_{3}(w, w)- \\
-\frac{\partial N_{t}}{\partial x} \frac{\partial w}{\partial x}-\frac{l^{2}}{2} \frac{\partial^{2}}{\partial x^{2}}\left(D_{0}(x) \frac{\partial^{2} w}{\partial x^{2}}\right)+ \\
+q+q_{*}=\rho h \frac{\partial^{2} w}{\partial t^{2}}+\varepsilon \rho h \frac{\partial w}{\partial t} \\
L_{1}(u, w)=\frac{\partial}{\partial x}\left(C_{0} \frac{\partial u}{\partial x}\right) \frac{\partial w}{\partial x} \\
L_{2}(w, w)=\frac{\partial}{\partial x}\left(C_{0}\left(\frac{\partial w}{\partial x}\right)^{2}\right) \frac{\partial w}{\partial x} \\
L_{3}(w, w)=\frac{\partial}{\partial x}\left(C_{1} \frac{\partial^{2} w}{\partial x^{2}}\right) \frac{\partial w}{\partial x}
\end{gathered}
$$

- нелинейные слагаемые,

$$
C_{i}(x)=\int_{-\frac{h}{2}}^{\frac{h}{2}}(\lambda+2 \mu) z^{i} d z, D_{0}(x)=\int_{-\frac{h}{2}}^{\frac{h}{2}} \mu d z .
$$

Температурное поле не задается, а определяется из решения уравнений теплопроводности

$$
\alpha_{t}\left(\frac{\partial^{2} T}{\partial x^{2}}+\frac{\partial^{2} T}{\partial z^{2}}\right)=f(x, z)
$$

с соответствующими краевыми условиями 1-го, 2-го, 3-го рода.

Для решения уравнения (14) применим метод вариационных итераций $[38,39]$ и его модификацию. Представим решение в виде $T(x, y)=X(x) \cdot Y(y)$. В качестве начального приближения одну из функций зададим произвольным образом, не удовлетворяя краевым условиям теплопроводности, и далее применим процедуру Бубнова-Галеркина в форме Л.В. Канторовича [40] и получим обыкновенные дифференциальные уравнения второго порядка, которые позволяют получить аналитическое решение, удовлетворив одному из краевых условий 1-3-го рода. Теперь полученное решение считается заданным, и строится процедура Бубнова-Галеркина в форме Канторовича уже по другой координате и т. д. Этот итерационный процесс очень быстро сходится и позволяет получить аналитическое решение уравнения теплопроводности (14). Такую же процедуру метода вариационных итераций можно применить для трехмерного уравнения теплопроводности. Теория такого подхода была разработана в [39].

В качестве примера рассмотрим прямоугольную в поперечном сечении $(b=1)$ балку с граничными условиями - жесткое защемление с обоих концов:

$$
w(0, t)=w(a, t)=u(0, t)=u(a, t)=\frac{\partial w(0, t)}{\partial x}=\frac{\partial u(0, t)}{\partial x}=0,
$$

с начальными условиями: $w(x, 0)=0, u(x, 0)=0, \frac{\partial w(x, 0)}{\partial t}=0, \frac{\partial u(x, 0)}{\partial t}=0$,

с краевыми условиями для уравнения теплопроводности:

$$
\begin{gathered}
T(x, z)=\text { const }, z=-h / 2,0 \leq x \leq a, \\
T(x, z)=0, z=h / 2,0 \leq x \leq a, \\
T(x, z)=0,-h / 2 \leq z \leq h / 2, x=a, \\
T(x, z)=0,-h / 2 \leq z \leq h / 2, x=0,
\end{gathered}
$$

где $t$ - время; $w, u$ - прогиб и функция перемещения по $0 x$ соответственно; $h$ - толщина нанобалки; $q$ параметр внешней равномерно распределенной нагрузки; $\rho$ - плотность материала; $a$ - длина балки; $l$ - размерно-зависимый параметр; $T$ - температура; $q_{*}=w_{n} *\left(\frac{2 \operatorname{rand}()}{\operatorname{randmax}+1.0}-1.0\right)-$ белый шум - это обобщённый стационарный случайный процесс $X(t)$ с постоянной спектральной плотностью.

Для учета физической нелинейности материала балок применяются деформационная теория пластичности и метод переменных параметров упругости. Согласно этому методу модуль упругости и коэффициент Пуассона связаны с модулями сдвига и деформации следующими соотношениями:

$$
E=\frac{9 K G}{3 K+G} .
$$

Объемная деформация $K$ считается постоянной и равной $1,94 G_{0}$. В деформационной теории модуль сдвига определяется по формуле

$$
G=\frac{1}{3} \frac{\sigma_{i}\left(e_{i}\right)}{e_{i}} .
$$

Диаграмма деформирования материала балок может иметь произвольный вид. В данной работе рассматривается чистый алюминий:

$$
\sigma_{i}=\sigma_{s}\left[1-\exp \left(-\frac{e_{i}}{e_{s}}\right)\right] .
$$

Интенсивность деформаций определяется выражением

$$
e_{i}=\left|\frac{2}{3} e_{x x}\right|=\frac{2}{3}\left|\frac{\partial u}{\partial x}+\frac{1}{2}\left(\frac{\partial w}{\partial x}\right)^{2}-z \frac{\partial^{2} w}{\partial x^{2}}\right| .
$$

Здесь учтено, что составляющая $e_{z z}$ находится из условия плоского напряженного состояния $\left(\sigma_{z z}=0\right)$ :

$$
e_{z z}=-\frac{v}{1-v} e_{x x} .
$$

Закон изменения нагрузки во времени и вдоль оси балки может быть произвольным.

\section{Метод решения уравнений движения}

Для решения системы нелинейных дифференциальных уравнений в частных производных (16) используется следующий алгоритм:

1. Сведение к задаче Коши осуществляется методом конечных разностей второго порядка точности по пространственной координате х. 
2. Решение задачи Коши по времени методом Ньюмарка. На каждом шаге по времени в точках $n_{x}, n_{z} \in \Omega$ (здесь $n_{x}, n_{z}-$ количество точек, на которое разбивается область $\Omega$ ) вычисляется интенсивность деформаций (18), интенсивность напряжений (17) определяется модуль упругости (15) и модуль сдвига (16) при известных значениях прогиба $w$ и перемещения $u$. Вычисляются переменные коэффициенты по толщине и длине, определенные интегралы для $\mathrm{C}_{i}, \mathrm{C}_{2}, \mathrm{D}_{0}$ вычисляются по формуле Симпсона. Так как шаг по времени очень мал, то строить итерационную процедуру переменных параметров упругости не следует. Для стационарных задач на каждом уровне нагрузки следует проводить итерационный процесс параметров упругости. Численный эксперимент это подтвердил.

\section{Численный эксперимент}

Приведем результаты численного исследования чувствительного элемента акселерометра для измерения параметров буровых скважин в виде геометриче-
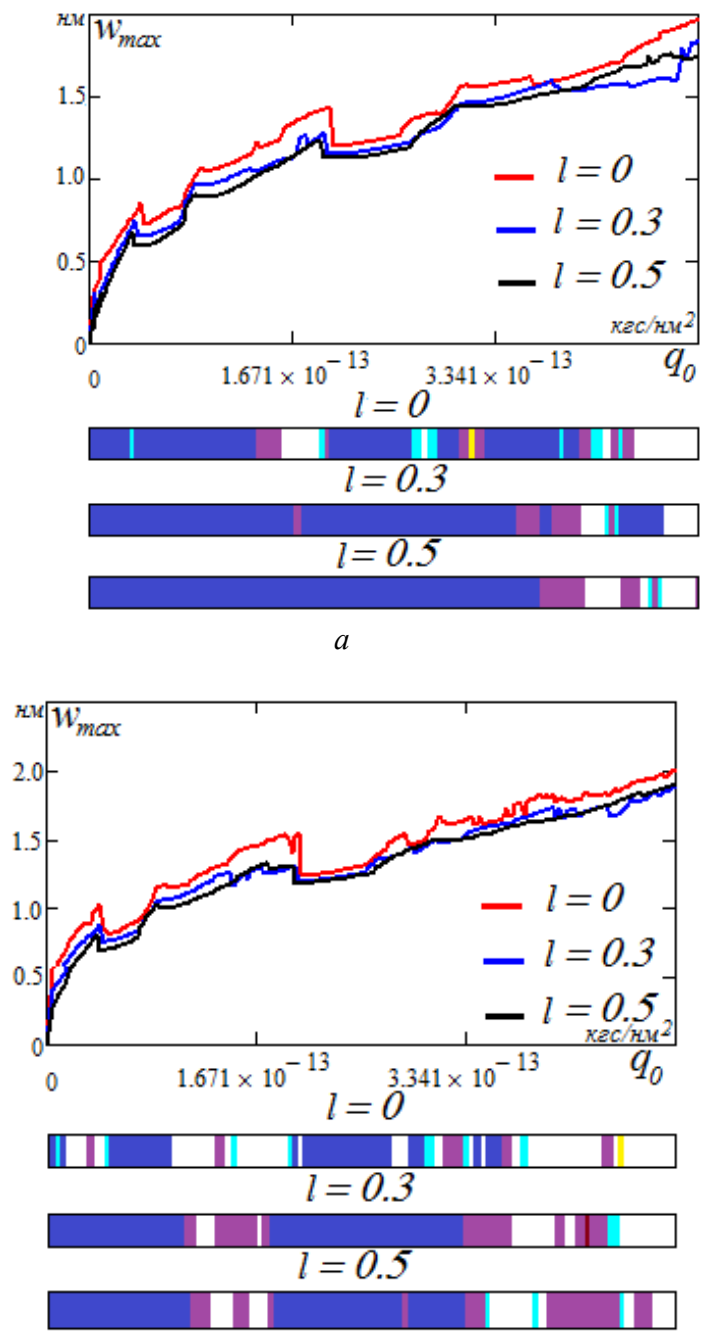

$c$ ски и физической нелинейной жестко защемленной нанобалки, находящейся в температурном поле, к которой приложена знакопеременная и шумовая нагрузка. Здесь $\omega_{p}=5$ - частота линейных собственных колебаний. Рассмотрена балка со следующими геометрическими и физическими параметрами: длина $a=400$ нм, толщина $h=4$ нм, $l=0 ; 0,3 ; 0,5$ нм. Были рассмотрены следующие случаи:

1) балка находится под действием знакопеременной нагрузки (рис. 2, a);

2) балка находится в аддитивном белом шуме и под действием знакопеременной нагрузки (рис. $2, b$ );

3) балка находится в температурном поле и под действием знакопеременной поперечной нагрузки (рис. 2, c);

4) балка находится в температурном поле, аддитивном белом шуме и под действием знакопеременной поперечной нагрузки (рис. $2, d$ ).
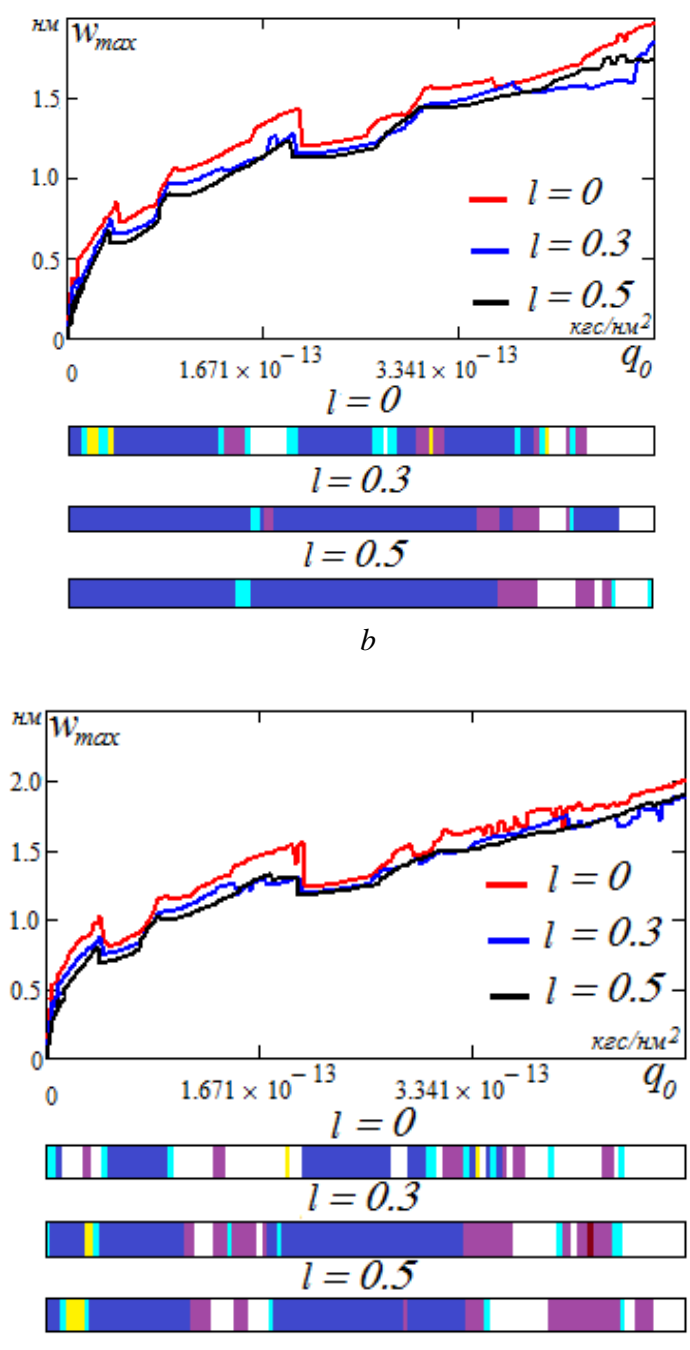

Условные обозначения

Гармонические колебания на частоте $\omega_{p}$ Гармонические колебания на частоте $\frac{\omega_{p}}{2^{n}}$
Колебания на двух независимых частотах

Колебания на двух независимых частотах и их линейных комбинациях 


\section{Xaoc}

Pис. 2 Шкалы характера колебаний и зависимости максимального прогиба в иентре балки от амплитуды нагрузки $w_{\text {max }}\left(q_{0}\right):$ a) температура $T=0^{\circ} \mathrm{C}$, интенсивность иума $\left.w_{n}=0 ; b\right)$ температура $T=0{ }^{\circ} \mathrm{C}$, интенсивность иума $w_{n}=5$; с) температура $T=100^{\circ} \mathrm{C}$, интенсивность иума $w_{n}=0$; d) температура $T=100^{\circ} \mathrm{C}$, интенсивность иума $w_{n}=5$

Fig. 2. Scales of the nature of oscillations and dependences of the maximum deflection on the amplitude of the load $w_{\text {max }}\left(q_{0}\right)$ a) temperature $T=0{ }^{\circ} \mathrm{C}$, noise intensity $\left.w_{n}=0 ; b\right)$ temperature $T=0{ }^{\circ} \mathrm{C}$, noise intensity $\left.w_{n}=5 ; c\right)$ temperature $T=100^{\circ} \mathrm{C}$, noise intensity $w_{n}=0 ; d$ ) temperature $T=100^{\circ} \mathrm{C}$, noise intensity $w_{n}=5$

При решении задач анализировались сигналы, фазовые портреты, сечение Пуанкаре, автокорреляционная функция, Фурье-спектр, знак старшего показателя Ляпунова во времени для каждой из балок в зависимости от величины размерно-зависимого параметра. На рис. 2 приведены шкалы характера колебаний и зависимости максимального прогиба от амплитуды нагрузки в центре балки $w_{\max }\left(q_{0}\right)$ для некоторых значений размерно-зависимого параметра $l=0 ; 0.3 ; 0.5$ (при $l=0$ без учета наноструктуры, $l=0,3 ; 0,5$ - учитывается наноструктура). Анализ численных результатов показал, что температурное и шумовое поле увеличивает области хаотических колебаний, зоны удвоения периода и колебаний на двух независимых частотах для упругих, изотропных, но неоднородных балок. С увеличением размернозависимого параметра увеличиваются зоны гармонических колебаний. При $l=0$ переход от гармонических колебаний к хаотическим происходит по сценарию Рюэля-Такенса-Ньюхауза, т. е. появляется независимая частота и линейные комбинации частоты возбуждения. При $l=0,3 ; 0,5$ хаотические колебания до $q_{0}=4,6 * 10^{-13} \mathrm{\kappa rc/м^{2 }}$ отсутсвуют, в то время как для $l=0$ такого не наблюдается.

\section{Заключение}

Построена математическая модель нелинейной динамики чувствительных элементов акселерометра для измерения параметров буровых скважин. Проведены численные эксперименты для чувствительных

\section{СПИСОК ЛИТЕРАТУРЫ}

1. Бойков В.И., Смирнов А.И. Построение модели пьезоактюатора с учетом нежесткости основания // Известия высших учебных заведений. Приборостроение. - 2019. - Т. 62. - № 4. С. 393-399.

2. Левицкий А.А., Маринушкин П.С. Проектирование микросистем. Программные средства обеспечения САПР. - Красноярск: Сибирский федеральный университет, 2010. - 156 с.

3. Галкина С.А., Барулина М.А. Влияние неизотропности материала на деформацию чувствительного элемента микромеханического акселерометра // Нано- и микросистемная техника. 2019. - T. 21. - № 1. - C. 14-23.

4. Лестев А.М., Фёдоров М.В., Евстафьев С.Д. Идентификация структуры шумов микромеханических инерциальных преобразователей параметров движения // Радиопромышленность. 2019. - Т. 29. - № 2. - С. 69-75.

5. Расчет угловой жесткости упругого элемента микромеханического акселерометра / М.Е. Калинкина, А.С. Козлов, Р.Я. Лабковская, О.И. Пирожникова, В.Л. Ткалич // Известия высших учебных заведений. Приборостроение. - 2019. - Т. 62. - № 6. С. 534-541.

6. Антонов Е.А., Меркурьев И.В., Подалков В.В. Влияние нелинейной жесткости упругих элементов на динамику двухмассового микромеханического гироскопа L-L-типа в режиме вынужденных колебаний // Вестник Томского государствен- элементов наноэлектромеханических систем, которые могут рассматриваться как гибкие упругие изотропные, но неоднородные нанобалки, находящиеся в стационарном температурном поле, под действием поперечной знакопеременной нагрузки и аддитивного белого шума. Математическая модель представляет собой систему дифференциальных уравнений в частных производных высокого порядка и высокой нелинейности. Проведен анализ устойчивости решения полученной системы дифференциальных уравнений, определен сценарии перехода от гармонических колебаний исследуемого объекта к хаотическому (сценарий Рюэля-Такенса-Ньюхауза).

Выявлено, что температурное и шумовое поля уменьшают нагрузку, при которой происходит переход в хаотическое состояние системы. Это существенно, если расчетная модель элемента прибора адекватно отражает условия его работы (т. е. учет неоднородности материала и физической нелинейности). Это указывает на то, что выбор расчетной схемы при расчете приборов НЭМС, применяемых в георазведке, чрезвычайно важен. Уменьшение чувствительного элемента прибора до наноразмера (учет размерно-зависимого параметра $l$ в математической модели) приводит к увеличению зон гармонических колебаний и, как следствие, к стабильной работе и долговечности работы прибора.

Работа выполнена при финансовой поддержке гранта РНФ № 19-19-00215.

ного университета. Математика и механика. - 2019. - № 57. C. 53-61.

7. Лестев А.М., Ефимовская А.В. О влиянии нелинейных факторов на динамику микромеханического гироскопа с двухмассовым чувствительным элементом // Известия высших учебных заведений. Приборостроение. - 2012. - Т. 55. - № 5. C. $40-46$.

8. Скважинные насосные установки для добычи нефти / В.Н. Ивановский, В.И. Дарищев, А.А. Сабиров, В.С. Каштанов, С.С. Пекин. - М.: Изд-во «Нефть и газ» РГУ нефти и газа им. И.М. Губкина, 2002. - 824 с

9. Котов А.А., Коротаев Б.А. Расчет бурильной колонны для бурения на акватории // Известия высших учебных заведений. Арктический регион. - 2017. - № 1. - С. 112-119.

10. Юнин Е.К., Турыгин Е.Ю. К вопросу оценки касательных напряжений, вызываемых крутильными автоколебаниями бурильной колонны // Строительство нефтяных и газовых скважин на суше и на море. - 2010. - № 6. - С. 6-13.

11. Дорофеев А.А. Исследование кремниевых наноэлектромеханических резонаторов на основе подвешенных наноструктур // Ломоносов 2019: Международная научная конференция студентов, аспирантов и молодых ученых. - М.: Московский государственный университет имени М.В. Ломоносова. 2019. С. 628-629. 
12. Хвесюк В.И., Скрябин А.С. Теплопроводность наноструктур // Теплофизика высоких температур. - 2017. - Т. 55 - № 3. C. 447-471.

13. Юров В.М., Гученко С.А., Лауринас В.Ч. Коэффициент теплопроводности наностуктур // Инновационная наука. - 2019. № 5. - С. 10-14.

14. Гринберг Я.С., Пашкин Ю.А., Ильичев Е.В. Наномеханические резонаторы // Успехи физических наук. - 2012. - Т. 182. № 4. - C. 407-436.

15. Strain gradient plasticity: theory and experiments / N.A. Fleck, G.M. Muller, M.F. Ashby, J.W. Hutchinson // Acta Metallurgica et Materialia. - 1994. - № 42. - P. 475-487.

16. Mazza E., Abel S., Dual J. Experimental determination of mechanical properties of $\mathrm{Ni}$ and Ni-Fe microbars // Microsystem Technologies. - 1996. - V. 2. - № 4. - P. 197-202.

17. Experiments and theory in strain gradient elasticity / D.C.C. Lam F. Yang, A.C.M. Chong, J. Wang, P. Tong // Journal of the Mechanics and Physics of Solids. - 2003. - № 51. - P. 1477-1508.

18. Ma Q., Clarke D.R. Size dependent hardness of silver single crystals // Journal of Materials Research. - 1995. - V. 10. - № 4. P. 853-863.

19. Engelman D.M. Membranes are more mosaic than fluid (review) // Nature. - 2005. - V. 438. - № 7068. - P. 578-580.

20. Cosserat E., Cosserat F. Théorie des corps déformables. - Paris Hermann et Fils, 1909. - 242 p.

21. Couple stress based strain gradient theory for elasticity / F. Yang, A.C.M. Chong, D.C.C. Lam, P. Tong // International Journal of Solids and Structures. - 2002. - № 39. - P. 2731-2743.

22. Chaotic dynamics of the size-dependent non-linear micro-beam model / A.V. Krysko, J. Awrejcewicz, S.P. Pavlov, M.V. Zhigalov, V.A. Krysko // Communications in Nonlinear Science and Numerical Simulation. - 2017. - № 50. - P. 16-28.

23. Park S.K., Gao X.L. Bernoulli-Euler beam model based on modified couple stress theory // Micromech Microeng. - 2006. V. 16. - № 11. - P. 2355-2359.

24. The size-dependent natural frequency of Bernoulli-Euler microbeams / Sh. Kong, Sh. Zhou, Zh. Nie, K. Wang // Internationa Journal of Engineering Science. - 2008. - № 46. - P. 427-437.

25. Rajabi F., Ramezani S. A nonlinear microbeam model based on strain gradient elasticity theory with surface energy // Archive of Applied Mechanics. - 2012. - № 82. - P. 363-376.

26. Дмитриев А.С. Тепловые процессы в наноструктурах. - М.: ИД МЭИ, 2012. - 302 с.

27. Дмитриев А.С. Введение в нанотеплофизику. - М.: БИНОМ, 2015. $-792 \mathrm{c}$.

28. Hadjesfandiari A.R. Size-dependent thermoelasticity // Latin American Journal of Solids and Structures - 2014 - No 11 P. 1679-1708.
29. Soboley S.L. Equations of transfer in non-local media // International Journal of Heat and Mass Transfer. - 1994. № 14. - P. 2175-2182.

30. Tzou D.Y. Macro-to-micro-scale heat transfer: the Lagging behavior. - New-Jersey: CRC Press, 1996. - 576 p.

31. Brock L.M. Reflection of transient plane step-stress waves: Some considerations of orthotropy and thermoelasticity // Journal of Mechanics of Materials and Structures. - 2011. - V. 6. - № 1-4. P. 87-104.

32. Хорстхемке В., Лефевр Р. Индуцированные шумом переходы: теория и применение в физике, химии и биологии. - М.: Мир, 1987. $-400 \mathrm{c}$

33. Lynch J.J., Nagele R.G. Flicker noise effects in noise adding radiometers // IEEE Transactions on Microwave Theory and Techniques. - 2011. - № 59. - P. 196-205.

34. Хаотическая динамика гибких замкнутых цилиндрических нанооболочек при локальном нагружении / О.А. Салтыкова, О.А. Афонин, Т.В. Яковлева, А.В. Крысько // Нелинейный мир. - 2018. - Т. 16. - № 5. - С. 3-15.

35. Параметрические колебания и контактное взаимодействие в компонентах микромеханических датчиков инерциальной информации / Т.В. Яковлева, А.В. Крысько, В.С. Кружилин, В.А. Крысько // Проблемы прочности и пластичности. 2018. - Т. 80. - № 1. - С. 63-71.

36. Новая методика определения истинности хаотической динамики чувствительных элементов микро- и наноэлектромеханических систем / О.А. Афонин, О.А. Салтыкова, И.В. Папкова, А.В. Крысько // Нелинейный мир. - 2018. - Т. 16. - № 5. C. 23-32.

37. Quantyfying chaos by various computational methods. P. 1: Vibrations of the Bernoulli-Euler beam subjected to periodic and colored noise / J. Awrejcewicz, A.V. Krysko, N.P. Erofeev, V. Dobriyan, M.A. Barulina, V.A. Krysko // Entropy. - 2018. V. 20. - № 3. - P. 170-183.

38. Математическое и компьютерное моделирование распределенных механических наноструктур / В.А. Крысько, С.П. Павлов, М.В. Жигалов, О.А. Салтыкова, А.В. Крысько. Саратов: Саратовский государственный технический университет им. Гагарина Ю.А., 2018. - 384 с.

39. Contact interaction of two rectangular plates made from different materials with an account of physical non-linearity / J. Awrejcewicz, V.A. Krysko, M.V. Zhigalov, A.V. Krysko // Nonlinear Dynamics. - 2018. - V. 91. - № 2. - P. 1191-1211.

40. Канторович Л.В., Крылов В.И. Приближенные методы высшего анализа. 5-е изд. - М.; Л.: Физматлит, 1962. - 708 с.

Поступила 14.11.2019 2.

\section{Информация об авторах}

Крысько В.А., доктор технических наук, профессор, заведующий кафедрой математики и моделирования, Саратовский государственный технический университет им. Ю.А. Гагарина.

Папкова И.В., кандидат физико-математических наук, доцент кафедры математики и моделирования, Саратовский государственный технический университет им. Ю.А. Гагарина; инженер, Национальный исследовательский Томский политехнический университет.

Яковлева T.B., кандидат физико-математических наук, доцент кафедры математики и моделирования, Саратовский государственный технический университет им. Ю.А. Гагарина; инженер, Национальный исследовательский Томский политехнический университет.

Захарова A.A., доктор технических наук, профессор кафедры информатики и программного обеспечения Брянского государственного технического университета; старший научный сотрудник, Национальный исследовательский Томский политехнический университет.

Жигалов М.В., доктор физико-математических наук, профессор кафедры математики и моделирования, Саратовский государственный технический университет им. Ю.А. Гагарина.

Крысько A.B., доктор физико-математических наук, главный научный сотрудник НОЦ «Математическое и компьютерное моделирование», Саратовский государственный технический университет им. Ю.А. Гагарина; инженер, Национальный исследовательский Томский политехнический университет. 


\title{
DYNAMICS OF A GEOMETRICALLY AND PHYSICALLY NONLINEAR SENSITIVE ELEMENT OF A NANOELECTROMECHANICAL SENSOR IN THE FORM OF AN INHOMOGENEOUS NANOBEAM IN THE TEMPERATURE AND NOISE FIELDS
}

\author{
Vadim A. Krysko1, \\ tak@san.ru \\ Irina V. Papkova1,2, \\ ikravzova@mail.ru \\ Tatiana V. Yakovleva1,2, \\ yan-tan1987@mail.ru \\ 1 Yuri Gagarin Saratov State Technical University, \\ 77, Politechnicheskaya street, Saratov, 410054, Russia. \\ 2 National Research Tomsk Polytechnic University, \\ 30, Lenin avenue, Tomsk, 634050, Russia. \\ 3 Bryansk State Technical University, \\ 7, 50 let Oktyabrya blvd, Bryansk, 241035, Russia.
}

\author{
Alena A. Zakharova3,2, \\ zaa@tu-bryansk.ru \\ Maksim V. Zhigalov ${ }^{1}$, \\ zhigalovm@yandex.ru \\ Anton V. Krysko1,2, \\ anton.krysko@gmail.com
}

The research relevance. Nanoelectromechanical systems, being highly sensitive sensors with small dimensions and reliable in operation, are increasingly used in the oil and gas industry for monitoring various processes in oil production, from exploration to enhanced oil recovery, as well as in well drilling, cleaning, fractionation and processing before decommissioning. One application example of nanoelectromechanical systems is seismic exploration. The use of nanoelectromechanical systems offers improved performance in addition to significant cost and time savings for a wide range of oil and gas industry technologies. With continuous monitoring capabilities, these technologies can become the foundation of smart deposits.

The main aim of the research is a construction of a mathematical model that most closely describes the sensitive element nonlinear dynamics of a nanoelectromechanical sensor under an alternating load action. For this, it is necessary to take into account the most common kinematic hypotheses, scale effects using the modified couple stress theory of elasticity, the nonlinear relationship between stresses and strains, the material inhomogeneity, noise and thermal fields. And also to examine the complex nonlinear oscillations nature and identify patterns of transition from harmonic to chaotic.

Objects: geometrically and physically nonlinear nanobeam, described by the kinematic model of the first approximation, which is affected by a uniformly distributed alternating transverse load with a harmonic component, the temperature field and additive external noise. Methods: variation methods, a second-order finite difference method for reducing the system of nonlinear partial differential equations to the Cauchy problem, the Newmark method for solving the Cauchy problem, the Birger method of variable elasticity parameters for solving a physically non-linear problem, the variation iteration method for obtaining an analytical solution of the two-dimensional heat equation. Results. The variation iterations method is used to obtain an analytical solution of thermal conductivity. An oscillations mathematical model for the sensitive element of the nanoelectromechanical sensor in the form of a size-dependent beam, on which a uniformly distributed transverse load with a harmonic component acts, is constructed. In addition to the variable load, the influence of the temperature field and additive external noise exposure were taken into account. The geometric nonlinearity is accepted according to Theodore von Karman theory (the relationship between deformations and displacements). To take into account the physical nonlinearity of the beam material, the deformation plasticity theory and the method of variable elasticity parameters are used. The motion equations of a mechanical system element, as well as the corresponding boundary and initial conditions, are derived from the Ostrogradsky-Hamilton principle based on a modified moment theory taking into account the Euler-Bernoulli hypothesis. It was revealed that the temperature and noise fields reduce the load at which the transition to the chaotic state of the system occurs. The transition from harmonic to chaotic oscillations occurs according to Ruelle-Takens-Newhouse scenario.

\section{Key words:}

Euler-Bernoulli nanobeam, nanoelectromechanical system, accelerometer for measuring borehole parameters, temperature and noise fields, modified couple stress theory of elasticity, finite difference and Newmark methods, chaotic oscillations of a nanoelectromechanical system.

The research was financially supported by the RSF grant no. 19-19-00215.

\section{REFERENCES}

1. Boykov V.I., Smirnov A.I. Building a piezo actuator model taking into account the nonrigidity of the base. Journal of Instrument Engineering, 2019, vol. 62, no. 4, pp. 393-399. In Rus.

2. Levitckiy A.A., Marinushkin P.S. Proektirovanie mikrosistem. Programmnye sredstva obespecheniya SAPR [Microsystem design. CAD software]. Krasnoyarsk, SibFU Publ., 2010. 156 p.

3. Galkina S.A., Barulina M.A. The effect of the non-isotropy of the material on the deformation of the sensitive element of the micromechanical accelerometer. Nano and microsystem technology, 2019, vol. 21, no. 1, pp. 14-23. In Rus.
4. Lestev A.M., Fedorov M.V., Evstafev S.D. Identifikatsiya struktury shumov mikromekhanicheskih inertsialnyhh preobrazovateley parametrov dvizheniya [Identification of the noise structure of micromechanical inertial converters of motion parameters]. Radiopromyshlennost, 2019, vol. 29, no. 2, pp. 69-75.

5. Kalinkina M.E., Kozlov A.S., Labkovskaya R.Ya., Pirozhnikova O.I. Tkalich V.L. Calculation of the angular stiffness of the elastic element of the micromechanical accelerometer. Journal of Instrument Engineering, 2019, vol. 62, no. 6, pp. 534-541. In Rus.

6. Antonov E.A., Merkurev I.V., Podalkov V.V. The influence of nonlinear rigidity of elastic elements on the dynamics of a two- 
mass L-L-type micromechanical gyroscope in the forced oscillation mode. Bulletin of the Tomsk state university. Mathematics and mechanics, 2019, no. 57, pp. 53-61. In Rus.

7. Lestev A.M., Efimovskaya A.V. On the influence of nonlinear factors on the dynamics of a micromechanical gyroscope with a two-mass sensitive element. Journal of Instrument Engineering, 2012, vol. 55, no. 5, pp. 40-46. In Rus.

8. Ivanovskiy V.N., Darishev V.I., Sabirov A.A., Kashtanov V.S., Pekin S.S. Skvazhinnye nasosnye ustanovki dlya dobychi nefti [Oil well pumping units]. Moscow, I.M. Gubkin Oil and gas RGU, 2002. $824 \mathrm{p}$.

9. Kotov A.A., Korotaev B.A. Calculation of a drill string for drilling in the water area. News of higher educational institutions. Arctic region, 2017, no. 1, pp. 112-119. In Rus.

10. Yunin E.K., Turygin E.Yu. K voprosu otcenki kasatelnykh napryazheniy, vyzyvaemykh krutilnymi avtokolebaniyami burilnoy kolonny [On the issue of internal shear caused by torsion self-vibrations of drill string. Construction of oil and gas wells on land and at sea, 2010, no. 6, pp. 6-13. In Rus.

11. Dorofeev A.A. Issledovanie kremnievykh nanoelektromekhanicheskikh rezonatorov na osnove podveshennykh nanostruktur [Study of silicon nanoelectromechanical resonators based on suspended nanostructures]. Lomonosov 2019. Mezhdunarodnaya nauchnaya konferentsiya studentvov, aspirantov i molodykh ucgenykh [International scientific conference of students and young scientists. Lomonosov 2019]. Moscow, Lomonosov Moscow State University Publ., 2019. pp. 628-629.

12. Khvesyuk V.I., Skryabin A.S. Teploprovodnost nanostruktur [Thermal conductivity of nanostructures]. Teplofizika vysokikh temperatur, 2017, vol. 55, no. 3, pp. 447-471.

13. Yurov V.M., Gurchenko S.A., Laurinas V.Ch. Koeffitsient teploprovodnosti nanostruktur [Thermal conductivity of nanostructures]. Innovatcionnaya nauka, 2019, no. 5, pp. 10-14.

14. Grinberg Ya.S., Pashkin Yu.A., Ilichev E.V. Nanomekhanicheskie rezonatory [Nanomechanical resonators]. Uspekhi fizicheskikh nauk, 2012, vol. 182, no. 4, pp. 407-436.

15. Fleck N.A., Muller G.M., Ashby M.F., Hutchinson J.W. Strain gradient plasticity: theory and experiments. Acta Metallurgica et Materialia, 1994, no. 42. pp. 475-487.

16. Mazza E., Abel S., Dual J. Experimental determination of mechanical properties of $\mathrm{Ni}$ and Ni-Fe microbars. Microsystem Technologies, 1996, vol. 2, no. 4, pp. 197-202.

17. Lam D.C.C., Yang F., Chong A.C.M., Wang J., Tong P. Experiments and theory in strain gradient elasticity. Journal of Materials Research, 2003, no. 51, pp. 1477-1508.

18. Ma Q., Clarke D.R. Size dependent hardness of silver single crystals. Journal of Materials Research, 1995, vol. 10, no. 4, pp. 853-863.

19. Engelman D.M. Membranes are more mosaic than fluid (review). Nature, 2005 , vol. 438 , no. 7068 , pp. 578-580.

20. Cosserat E., Cosserat F. Théorie des corps déformables [Theory of deformed elements]. Paris, Hermann et Fils, 1909. 242 p.

21. Yang F., Chong A.C.M., Lam D.C.C., Tong P. Couple stress based strain gradient theory for elasticity. International Journal of Solids and Structures, 2002, no. 39, pp. 2731-2743.

22. Krysko A.V., Awrejcewicz J., Pavlov S.P., Zhigalov M.V., Krysko V.A Chaotic dynamics of the size-dependent non-linear micro-beam model. Communications in Nonlinear Science and Numerical Simulation, 2017, no. 50, pp. 16-28.
23. Park S.K., Gao X.L. Bernoulli-Euler beam model based on a modified couple stress theory. Micromech Microeng, 2006, vol. 16, no. 11, pp. 2355-2359.

24. Kong Sh., Zhou Sh., Nie Zh., Wang K. The size-dependent natural frequency of Bernoulli-Euler micro-beams. International Journal of Engineering Science, 2008, no. 46, pp. 427-37.

25. Rajabi F., Ramezani S. A nonlinear microbeam model based on strain gradient elasticity theory with surface energy. Archive of Applied Mechanics, 2012, no. 82, pp. 363-376.

26. Dmitriev A.S. Teplovye protsessy $v$ nanostrukturakh [Thermal processes in nanostructures]. Moscow, MEI Publ. house, 2012. 302 p.

27. Dmitriev A.S. Vvedenie v nanoteplofiziku [Introduction to Nanothermophysics]. Moscow, BINOM Publ., 2015. 792 p.

28. Hadjesfandiari A.R. Size-dependent thermoelasticity. Latin American Journal of Solids and Structures, 2014, no. 11, pp. 1679-1708.

29. Soboley S.L. Equations of transfer in non-local media. International Journal of Heat and Mass Transfer, 1994, no. 14, pp. 2175-2182.

30. Tzou D.Y. Macro-to-micro-scale heat transfer: the Lagging behavior. New Jersey, CRC Press, 1996.576 p.

31. Brock L.M. Reflection of transient plane step-stress waves: Some considerations of orthotropy and thermoelasticity. Journal of Mechanics of Materials and Structures, 2011, vol. 6, no. 1-4, pp. 87-104.

32. Horsthemke W., Lefever R. Noise-induced transitions: theory and applications in physics, chemistry and biology. Berlin, Heidelberg, New York, Tokyo, Springer-Verlag, 1984. $400 \mathrm{p}$.

33. Lynch J.J., Nagele R.G. Flicker noise effects in noise adding radiometers. IEEE Transactions on Microwave Theory and Techniques, 2011, no. 59, pp. 196-205.

34. Saltykova O.A., Afonin O.A., Yakovleva T.V., Krysko A.V. The chaotic dynamics of flexible closed cylindrical nanoshells under local loading]. Non-linear world, 2018, vol. 16, no. 5, pp. 3-15. In Rus.

35. Yakovleva T.V., Krysko A.V., Kruzhilin V.S., Krysko V.A. Parametric oscillations and contact interaction in the components of micromechanical sensors of inertial information. Strength and ductility problems, 2018, vol. 80, no. 1, pp. 63-71. In Rus.

36. Afonin O.A., Saltykova O.A., Papkova I.V., Krysko A.V. A new method for determining the truth of the chaotic dynamics of sensitive elements of micro- and nanoelectromechanical systems. Nonlinear world, 2018, vol. 16, no. 5, pp. 23-32. In Rus.

37. Awrejcewicz J., Krysko A.V., Erofeev N.P., Dobriyan V., Barulina M.A., Krysko V.A. Quantyfying chaos by various computational methods. P. 1: Vibrations of the Bernoulli-Euler beam subjected to periodic and colored noise. Entropy, 2018, vol. 20, no. 3, pp. 170-183.

38. Krysko V.A., Pavlov S.P., Zhigalov M.V., Saltykova O.A., Krysko A.V. Matematicheskoe i kompyuternoe modelirovanie raspredelennykh mekhanicheskikh nanostruktur [Mathematical and computer modeling of distributed mechanical nanostructures]. Saratov, Yuri Gagarin Saratov State Technical University, 2018. 384 p.

39. Awrejcewicz J., Krysko V.A., Zhigalov M.V., Krysko A.V. Contact interaction of two rectangular plates made from different materials with an account of physical non-linearity. Nonlinear Dynamics, 2018, vol. 91, no. 2, pp. 1191-1211.

40. Kantorovich L.V., Krylov V.I. Priblizhennye metody vysshego analiza [Approximate methods of higher analysis]. Moscow, Leningrad, Fizmatlit Publ., 1962. 708 p.

Received: 14 November 2019.

\section{Information about the authors}

Vadim A. Krysko, Dr. Sc., professor, head of Mathematics and Modeling Department, Yuri Gagarin Saratov State Technical University.

Irina V. Papkova, Cand. Sc., associate professor, Yuri Gagarin Saratov State Technical University; engineer, National Research Tomsk Polytechnic University.

Tatiana V. Yakovleva, Cand. Sc., associate professor, Yuri Gagarin Saratov State Technical University; engineer, National Research Tomsk Polytechnic University..

Alena A. Zakharova, Dr. Sc., professor, Bryansk State Technical University; senior Researcher, National Research Tomsk Polytechnic University

Maksim V. Zhigalov, Dr. Sc., professor, Yuri Gagarin Saratov State Technical University.

Anton V. Krysko, Dr. Sc., professor, Yuri Gagarin Saratov State Technical University; engineer, National Research Tomsk Polytechnic University. 